\title{
Guillain-Barré syndrome following the first dose of Pfizer-BioNTech COVID-19 vaccine: case report and review of reported cases
}

\author{
Nadia Bouattour ${ }^{1,2}(\mathbb{0})$ Olfa Hdiji ${ }^{1,2} \cdot$ Salma Sakka ${ }^{1,2} \cdot$ Emna Fakhfakh $^{1,2} \cdot$ Khadija Moalla $^{1,2} \cdot$ Sawsan Daoud ${ }^{1,2}$. \\ Nouha Farhat ${ }^{1,2} \cdot$ Mariem Damak $^{1,2} \cdot$ Chokri Mhiri $^{1,2}$
}

Received: 6 October 2021 / Accepted: 10 November 2021 / Published online: 18 November 2021

(c) Fondazione Società Italiana di Neurologia 2021

\begin{abstract}
Background Since the SARS-CoV-2 pandemic has started in December 2019, millions of people have been infected all over the world. Vaccination is the most efficient tool to end this pandemic, but vaccine surveillance is necessary to identify side effects. Some studies have shown that neurological complications after COVID-19 vaccination are rare and dominated by demyelinating disease.

Case presentation We present a case of a 67-year-old man who presented 7 days following his first dose of Pfizer-BioNTech COVID-19 vaccine a rapidly progressive ascending muscle weakness. The diagnosis of Guillain-Barré syndrome (GBS) was confirmed according to the clinical features, the albumino-cytological dissociation in the cerebrospinal fluid, and the electroneuromyography findings. The workup for all known infections associated with immune-mediated GBS was negative. The patient received treatment with intravenous immunoglobulin. Neurological examination 1 month after discharge showed full recovery and he regained his baseline functional status.

Conclusions As far as we know, this is the first reported case in Tunisia. Although extremely rare, neurologists should remain vigilant for acute inflammatory demyelinating polyradiculoneuropathy after COVID-19 vaccination.
\end{abstract}

Keywords Guillain-Barré syndrome (GBS) · COVID-19 vaccine · SARS-CoV-2 · Neurological complications

\section{Introduction}

Guillain-Barré syndrome (GBS) is the most common acute immune-mediated polyradiculoneuropathy in the world [1]. The classical clinical presentation of GBS is a bilateral weakness with hyporeflexia or areflexia with or without sensory symptoms [2]. The cause of GBS is unknown; it is believed that an autoimmune response plays a role in the pathogenesis of this disease [2]. The suggested pathophysiology is molecular mimicry following respiratory or gastrointestinal infections and extremely rare following vaccination [3]. Since the pandemic was caused by COVID-19, several vaccines were approved by the food and drug administration

Nadia Bouattour

bouattour_nadia@yahoo.fr

1 Neurology's Department, Habib Bourguiba Hospital, Ferdaous Street, 3029 Sfax, Tunisia

2 Laboratory of Neurogenetics, Parkinson's Disease and Cerebrovascular Disease (LR-12-SP-19), University of Sfax, Sfax, Tunisia to control this pandemic and many side effects were reported ranging from fatigue, fever, and myalgia to more serious complications [3, 4]. We herein report a case of a patient who developed GBS 7 days after receiving the first dose of Pfizer-BioNTech COVID-19 vaccine. We report this case to increase awareness of acute inflammatory demyelinating polyradiculoneuropathy (AIDP) as a possible side effect of the COVID-19 vaccine and to understand if this syndrome is associated with a specific type of vaccine by an exhaustive reviewing of the literature.

\section{Case report}

A 67-year-old male, with a medical history of type II diabetes, was admitted for a weakness of the four limbs in July 2021. He presented 4 days before his admission complaints of progressive ascending weakness. He had received his first dose of COVID-19 (Pfizer-BioNTech) 7 days before presentation. He denied any history of recent fever, gastrointestinal, or upper respiratory tract illness. He denied also contracting 
COVID-19 infection. At physical examination on admission, his blood pressure was $124 / 80 \mathrm{~mm} \mathrm{Hg}$, his pulse was 76 bat/ min, and his body temperature was $37.3{ }^{\circ} \mathrm{C}$. Neurological examination showed a flaccid tetraparesis predominating in the lower limbs and weakness in distal lower limbs for foot and toes dorsiflexors. His upper limb power was a medical research council (MRC) grade 4/5 and his lower limb power was MRC grade 3/5. There was a generalized areflexia, with intact superficial and vibratory sensation. Examination of the cranial nerves was normal. No bowel or bladder dysfunction was reported.

Routine blood tests revealed a hemoglobin rate of 12.4 $\mathrm{g} / \mathrm{dL}$, a platelet count of $361000 / \mu \mathrm{L}$, and a white blood cell count of $6780 / \mu \mathrm{L}$. He had a C-reactive protein of $4 \mathrm{mg} / \mathrm{dL}$.

Electroneuromyography performed 1 day after admission showed typical features of AIDP. Reviewing the neurophysiological criteria of Rajabally et al. [5], there is a prolonged $F$ response in the left median and left tibial nerves. Table 1 summarizes the results of the electroneuromyography.

Lumbar puncture revealed an albumino-cytological dissociation with $0.8 \mathrm{~g} / \mathrm{L}$ of protein, $4 \mathrm{WBC} / \mathrm{mm}^{3}$, and normoglucorrachia. Cerebrospinal fluid (CSF) cytology was unremarkable. Extensive infectious and inflammatory workup of serum and CSF which included HIV antibodies, hepatitis $\mathrm{B}$ and $\mathrm{C}$ serologies, Campylobacter jejuni serology, Lyme titers, CMV titers, EBV titers, and anti-nuclear antibodies were all negative. Ganglioside antibodies GM1, GD1a, GD1b, GQ1b, and GM2 were negative. COVID-19 PCR from a nasopharyngeal swab was negative.

Based on the previous workup, and according to the Brighton criteria [6], the diagnosis of GBS was made with the highest level of diagnostic certainty, and an intravenous immunoglobulin (IVIG) treatment was started. He was given IVIG in the standard recommended dose $(0.4 \mathrm{~g} / \mathrm{kg} / \mathrm{day})$ for 5 days with rehabilitation. After 1 week of hospitalization, his strength began to improve. He was seen in the neurology clinic, outpatient department of the hospital 4 weeks after discharge, where neurological examination showed full recovery and he regained his baseline functional status.

\section{Discussion}

This paper reported a case of GBS in a patient who recently received the Pfizer-BioNTech vaccine. This vaccine is a synthetic messenger RNA vaccine (mRNA). Inside the human body, mRNA enters the human cell and produces the spike protein found on the surface of the virus. Our bodies recognize this protein as an invader and produce antibodies against it [4]. In some cases, this immune response can trigger autoimmune processes that lead to the production of antibodies against the myelin and cause GBS. This syndrome is an immune-mediated syndrome that involves a
Table 1 Summary of electromyography and nerve conduction studies

\begin{tabular}{|c|c|c|c|}
\hline Tests & Normal value & Right & Left \\
\hline \multicolumn{4}{|l|}{ Motor nerve conduction } \\
\hline \multicolumn{4}{|l|}{ DML (ms) } \\
\hline Median & $\leq 3.8$ & 4.3 & 4.1 \\
\hline Ulnar & $\leq 3.2$ & 2.2 & 2.5 \\
\hline Peroneal & $\leq 5$ & 4.9 & 5.6 \\
\hline Tibial & $\leq 4.5$ & 5.7 & 6.5 \\
\hline F waves (ulnar) & $\leq 30$ & 33.5 & 34.3 \\
\hline F waves (median) & $\leq 30$ & 28.8 & $\underline{37.5}$ \\
\hline F waves (tibial) & $\leq 50$ & 55.3 & $\underline{61.3}$ \\
\hline \multicolumn{4}{|l|}{ CMAP (mV) } \\
\hline \multicolumn{4}{|l|}{ Median } \\
\hline Wrist & $\geq 6$ & 0.7 & 1.2 \\
\hline \multicolumn{4}{|l|}{ Ulnar } \\
\hline Wrist & $\geq 6$ & 1.2 & 3.9 \\
\hline Below elbow & & 1.7 & 3 \\
\hline \multirow[t]{2}{*}{ Above elbow } & & & \\
\hline & & $\begin{array}{l}\text { Proximal } \\
\text { conduction } \\
\text { block }\end{array}$ & $\begin{array}{l}\text { Proximal } \\
\text { conduction } \\
\text { block }\end{array}$ \\
\hline Peroneal & $\geq 3$ & 2.5 & 3.5 \\
\hline Tibial & $\geq 6$ & 0.2 & 0.4 \\
\hline \multicolumn{4}{|l|}{$\operatorname{MCV}(\mathrm{m} / \mathrm{s})$} \\
\hline Median & $\geq 45$ & 57.6 & 51.6 \\
\hline Ulnar & $\geq 45$ & 55.3 & 56 \\
\hline Peroneal & $\geq 42$ & 42.6 & 41 \\
\hline Tibial & $\geq 42$ & 40.3 & 45.4 \\
\hline \multicolumn{4}{|c|}{ Sensory nerve conduction } \\
\hline \multicolumn{4}{|l|}{ SNAP (mV) } \\
\hline Ulnar & $\geq 10$ & 13 & 14 \\
\hline Median & $\geq 15$ & 7 & 7.5 \\
\hline Radial & $\geq 15$ & 11 & 15 \\
\hline Sural & $\geq 10$ & 6.4 & 11 \\
\hline Musculocutaneous & $\geq 10$ & 19 & 15 \\
\hline \multicolumn{4}{|l|}{$\operatorname{SCV}(\mathrm{m} / \mathrm{s})$} \\
\hline Ulnar & $\geq 45$ & 56.3 & 57.8 \\
\hline Median & $\geq 45$ & 43.8 & 48.6 \\
\hline Radial & $\geq 45$ & 64.6 & 62.4 \\
\hline Sural & $\geq 40$ & 42 & 45.5 \\
\hline Musculocutaneous & $\geq 40$ & 54.3 & 57.4 \\
\hline
\end{tabular}

$D M L$, distal motor latency; $C M A P$, compound muscle action potential; $M C V$, motor nerve conduction velocity; $S N A P$, sensory nerve action potential; $S C V$, sensory conduction velocity; values marked in bold are above or below normal; values marked in bold underlined meet Rajabally's criteria

variety of demyelinating conditions: AIDP like in our case, acute motor-sensory axonal neuropathy (AMSAN), acute motor axonal neuropathy (AMAN), and Miller Fisher syndrome [7].

The immunological pathophysiology of GBS was reinforced by many reported cases following vaccination against 


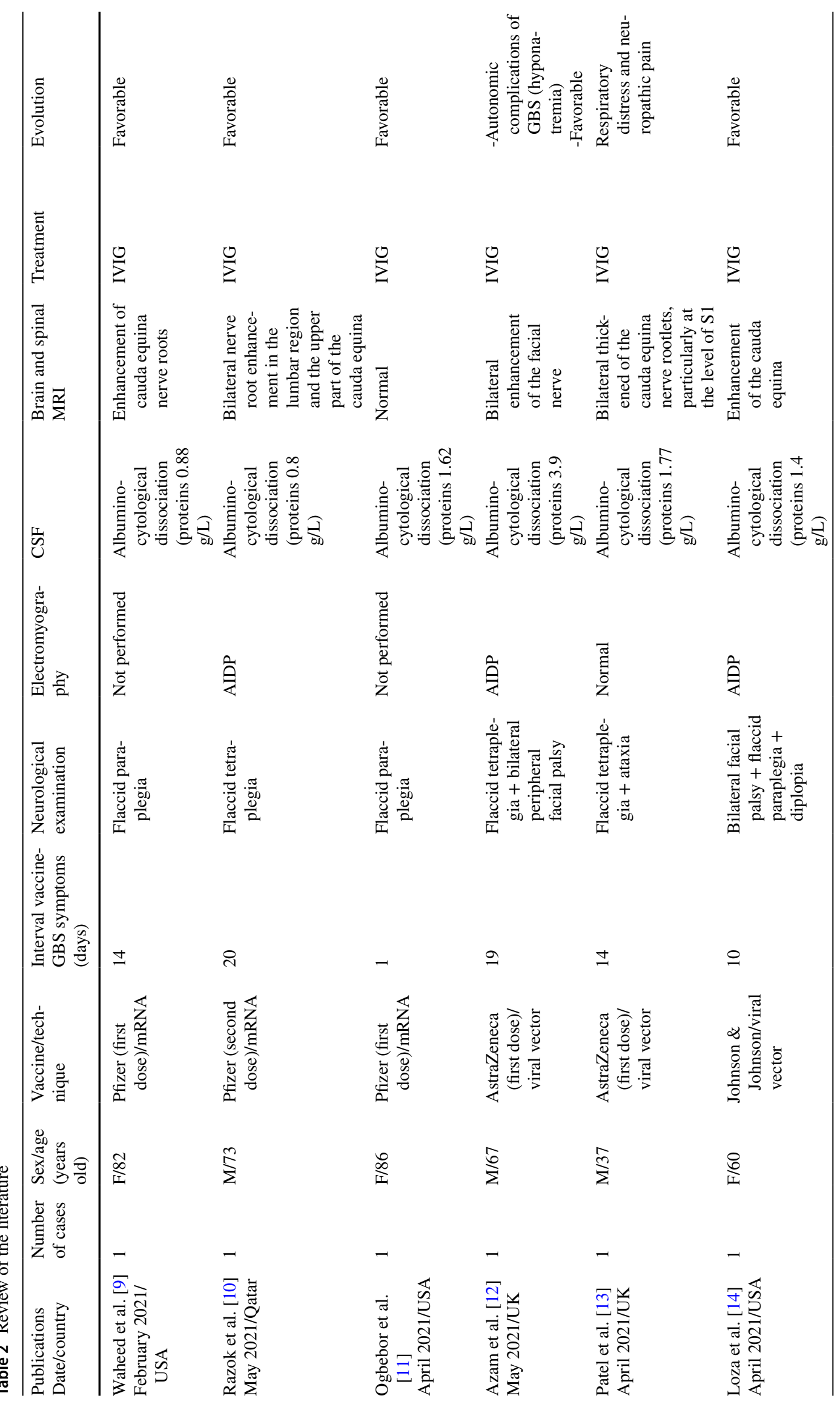




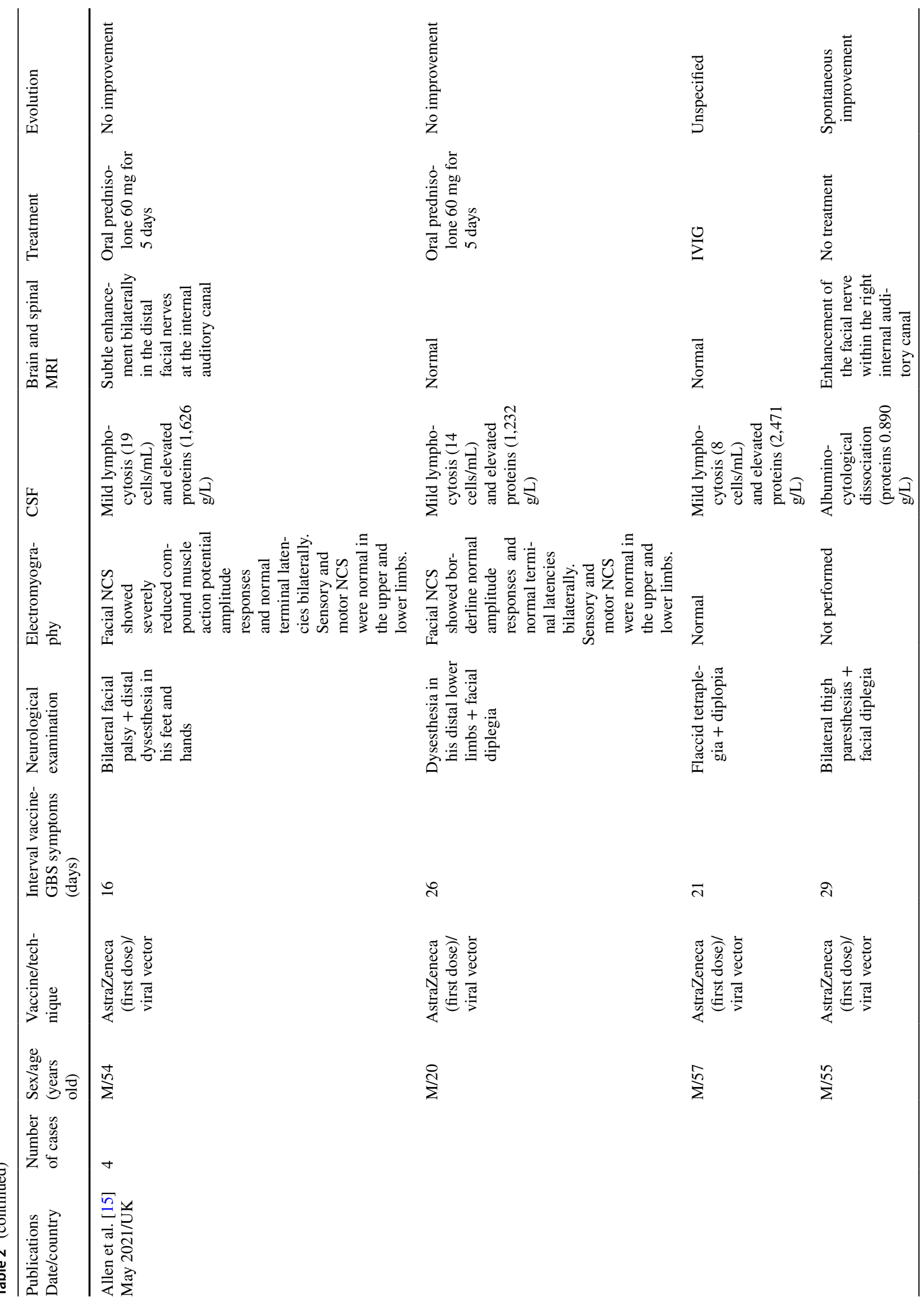




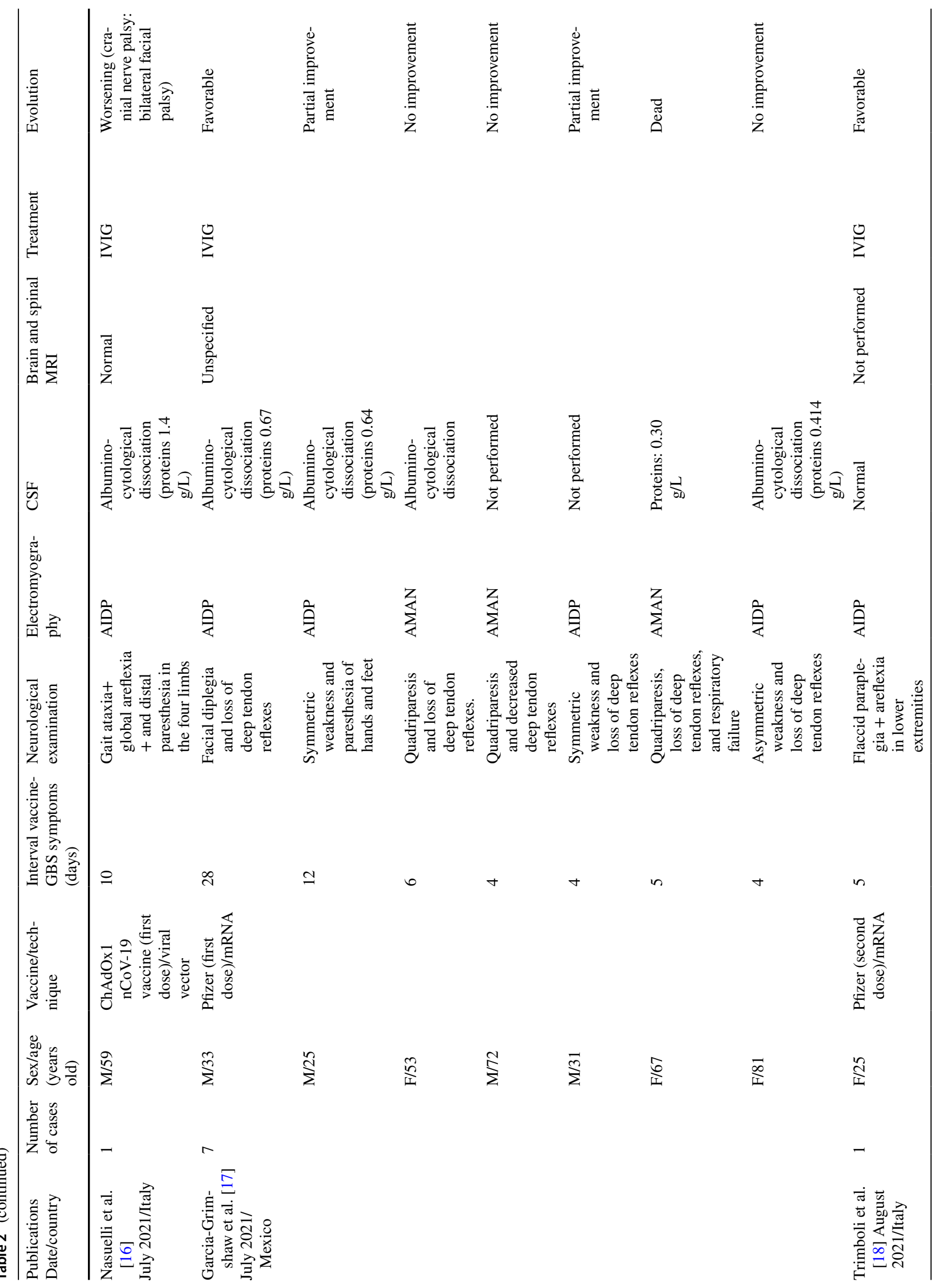




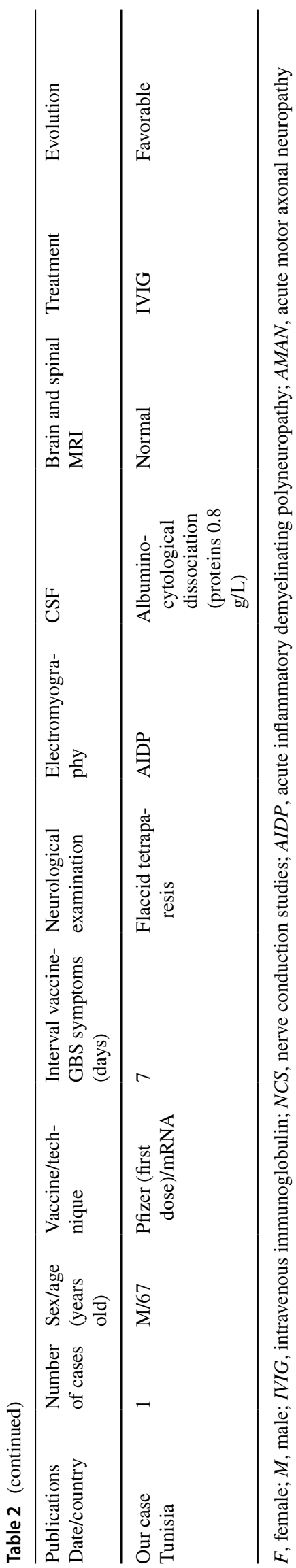

multiple pathogens. The influenza vaccine was the most offending, also hepatitis B and A, tetanus, and polio vaccines can cause GBS [8]. The first case of GBS following COVID-19 vaccination was reported by Waheed et al. [9] in February 2021 in the USA, in a 82-year-old female, 14 days after the first dose of Pfizer-BioNTech.

Reviewing the literature, at the time of writing this paper, 19 cases of GBS after COVID-19 vaccination were reported in the world (Table 2). All patients described in the literature had not a history of COVID-19 or current infection, as in our patient. Many types of vaccines with different mechanisms of action have been implicated in the development of GBS (Table 2): eleven cases after Pfizer-BioNTech vaccine, six cases after AstraZeneca vaccine, one case after ChAdOx1 nCoV-19 vaccine, and one case after Johnson \& Johnson vaccine. All reported cases presented GBS after receiving the first dose of COVID-19 vaccine, only one case after the second dose.

Our patient developed, 7 days after receiving the first dose of Pfizer-BioNTech vaccine, as far as our known this is the first reported case in Tunisia. However, despite a relatively large number of reported cases of GBS in postvaccination, a temporal association is a possibility and a definite causal association was not confirmed considering the inability to prove that relation on a molecular basis. The classical clinical manifestation of GBS is bilateral symmetric weakness and decreased deep tendon reflexes. The CSF analysis demonstrates albumin-cytological dissociation, like the findings in our patient and electrophysiological studies mainly showing AIDP. The treatment of GBS is based on plasma exchange (PE) or IVIG. Our patient and the majority of cases described in the literature underwent IVIG, because it is easier to manage than PE and has substantially fewer complications, only 2 cases were treated by prednisolone (Table 2).

\section{Conclusion}

We describe a case of GBS following vaccination against SARS-COV-2. We report this case to increase awareness of GBS as a possible complication of this vaccine, but further extensive studies are required to adequately determine the link between vaccination and GBS.

\section{Declarations}

Conflict of interest The authors declare no competing interests. 


\section{References}

1. Sejvar JJ et al (2011) Population incidence of Guillain-Barré syndrome: a systematic review and meta-analysis. Neuroepidemiology 36(2):123-133

2. Haber P et al (2009) Vaccines and Guillain-Barre syndrome. Drug Safety 32(4):309-323

3. Kim JH, Marks F, Clemens JD (2021) Looking beyond COVID-19 vaccine phase 3 trials. Nature medicine 27(2):205-211

4. Polack FP, et al. (2020) Safety and efficacy of the BNT162b2 mRNA Covid-19 vaccine. New England Journal of Medicine

5. Rajabally YA et al (2015) Electrophysiological diagnosis of Guillain-Barré syndrome subtype: could a single study suffice? Journal of Neurology. Neurosurgery \& Psychiatry 86(1):115-119

6. Fokke $\mathrm{C}$ et al (2014) Diagnosis of Guillain-Barré syndrome and validation of Brighton criteria. Brain 137(1):33-43

7. Willison HJ, Jacobs BC, van Doorn PA (2016) Guillain-Barre syndrome. The Lancet 388(10045):717-727

8. Souayah N et al (2011) Guillain-Barré syndrome after Gardasil vaccination: data from vaccine adverse event reporting system 2006-2009. Vaccine 29(5):886-889

9. Waheed, S., et al. (2021) Neurological complications of COVID19: Guillain-Barre syndrome following Pfizer COVID-19 vaccine. Cureus, 13(2)

10. Razok A, et al. (2021) Post-COVID-19 vaccine Guillain-Barré syndrome; first reported case from Qatar. Authorea Preprints
11. Ogbebor $\mathrm{O}$ et al (2021) Guillain-Barré syndrome following the first dose of SARS-CoV-2 vaccine: a temporal occurrence, not a causal association. IDCases 24:e1143

12. Azam S, Khalil A, Taha A (2021) Guillain-Barré syndrome in a 67-year-old male post COVID-19 vaccination (Astra Zeneca). American Journal of Medical Case Reports 9(8):424-427

13. Patel SU et al (2021) Guillain-Barre syndrome following the first dose of the chimpanzee adenovirus-vectored COVID-19 vaccine, ChAdOx1. BMJ Case Reports CP 14(4):e242956

14. Loza AMM et al (2021) Guillain-Barré syndrome in the placebo and active arms of a COVID-19 vaccine clinical trial: temporal associations do not imply causality. Neurology 96(22):1052-1054

15. Allen, C.M., et al. (2021) Guillain-Barré syndrome variant occurring after SARS-CoV-2 vaccination. Annals of Neurology

16. Nasuelli NA et al (2021) A case of acute demyelinating polyradiculoneuropathy with bilateral facial palsy after ChAdOx1 nCoV-19 vaccine. Neurological Sciences:1-3

17. García-Grimshaw M, et al. (2021) Guillain-Barré syndrome is infrequent among recipients of the BNT162b2 mRNA COVID-19 vaccine. Clinical Immunology, p. 108818

18. Trimboli M, et al. (2021) Guillain-Barré syndrome following BNT162b2 COVID-19 vaccine. Neurological Sciences, p. 1-2

Publisher's note Springer Nature remains neutral with regard to jurisdictional claims in published maps and institutional affiliations. 